\title{
Common complications with implants and implant prostheses
}

\author{
What complications are associated with dental implants?
}

\author{
Goodacre CJ, Bernal G, Rungcharassaeng K, Kan JY. Clinical \\ complications with implants and implant prostheses. J Prosthet \\ Dent 2003; 90:121-132
}

Data sources English language articles were sourced in MEDLINE. Study selection To be included in the review, published articles must have presented clinical data that identified the number of implants/ prostheses being evaluated, how long they had been in place, and how many were affected by complications.

Data extraction and synthesis Publications were grouped according to each category of complication (surgical, implant loss, bone loss, peri-implant soft tissue, mechanical and aesthetic/phonetic). The types of complications in each category were identified. The raw data for a particular complication were combined and a mean incidence of complications was calculated. The mean values for each complication were compared for the purpose of establishing a trend, allowing ranking of complications. For a specific complication to be included in this, three or more studies must have reported data related to the incidence of that particular complication.

Results The most common implant complications identified are shown in Table 1.

\section{Commentary}

Caveat Lector! This paper is registered as a meta-analysis in Medline. Meta-analyses should always be considered with caution because the quality of a meta-analysis will never be better than the quality of the independent studies that formed the basis of the statistical synthesis. If one identifies a possible selection bias of studies because of an unclear description of inclusion and exclusion criteria, an unclear description of the process to identify studies and lack of appraisal of the methodological quality of the included studies, there are good reasons to suspect that the conclusions may be invalid.

The authors here base their findings on approximately 210 papers published from 1981 to 2001, of which several are case descriptions of some very unusual adverse events. The merits of such case descriptions should not be undervalued, as they may be indicators of under-reporting in other studies. On the other hand, since there were an additional 600 clinical studies published within this time period (not counting case descriptions) one has to ask, what is so distinguishing about the selected studies? It is disquieting that only three out of 16 published clinical studies over 10 years, and only two out of eight studies with 15 years of observation were included;

Table 1. Most common implant complications ( $10 \%$ or greater incidence).

\begin{tabular}{|c|c|c|c|c|c|c|}
\hline \multirow[t]{2}{*}{ Complication } & \multicolumn{2}{|c|}{ Prostheses } & \multicolumn{2}{|c|}{ Implants } & \multicolumn{2}{|c|}{ Patients } \\
\hline & $N$ & Proportion (\%) & $N$ & Proportion (\%) & $N$ & Proportion (\%) \\
\hline Overdenture clip/attachment loosening & $113 / 376$ & 30 & & & & \\
\hline Resin veneer fracture/fixed partial dentures & $144 / 663$ & 22 & & & & \\
\hline Implant loss with maxillary overdentures & & & $206 / 1103$ & 19 & & \\
\hline Overdenture relines needed & $114 / 595$ & 19 & & & & \\
\hline Overdenture fracture & $69 / 570$ & 12 & & & & \\
\hline Opposing prosthesis fracture & $20 / 168$ & 12 & & & & \\
\hline Implant loss in smokers & & & $178 / 1668$ & 11 & & \\
\hline Implant loss with short implants ( $\leqslant 10 \mathrm{~mm})$ & & & $272 / 2754$ & 10 & & \\
\hline Implant loss with maxillary fixed complete dentures & & & $443 / 4559$ & 10 & & \\
\hline Aesthetic complication with prostheses & $47 / 493$ & 10 & & & & \\
\hline
\end{tabular}

*Type IV bone has a thin cortex and poor medullary strength with low trabecular density.

Conclusions It was not possible to calculate an overall incidence of complications for implant prostheses because there were not multiple clinical studies that simultaneously evaluated all or most of the categories of complications. Although the implant data had to be obtained from different studies, they do indicate a trend toward a greater incidence of complications with implant prostheses than single crowns, fixed partial dentures, all-ceramic crowns, resin-bonded prostheses, and posts and cores.

Address for correspondence: Dr Charles J Goodacre, Loma Linda University, School of Dentistry, Office of the Dean, Loma Linda CA 92350, USA. E-mail: cgoodacre@sd.Ilu.edu also, of the 20 largest studies, all including more than 1000 implants, only seven were included. The data from these studies would provide a more realistic picture of the incidence of failures than the data from the short-term clinical studies.

A striking error is that the term "incidence" is consistently applied incorrectly in both this and the matching paper focusing on fixed prostheses. ${ }^{1}$ Incidence always includes, by definition, a specified period of time, eg, months or years. Nowhere in the text are the complication rates related to the observation time, which more or less makes all the estimated "incidences" meaningless. For example, in the first section of the introduction it is suggested that, "the lowest incidence of complications" among fixed prostheses is 
observed for all-ceramic crowns compared with other prostheses. ${ }^{1}$ Such conclusions emerge when data from short-term trials of fullceramic crowns are added uncritically to data from long-term conventional prostheses. It would have made a difference if the complication rates in both papers had been linked to length of follow-up 2, 5 or 10 years, for example.

Because of the methodology of this paper, it is probable that the reported complication "incidences" are inflated. This paper suggests that neurosensory disturbances occur for $7 \%$ of all placed implants and that $19 \%$ of all maxillary implants supporting overdentures will come loose. Clearly, these numbers are not reflected in longitudinal clinical studies nor in systematic reviews on the topic., ${ }^{2,3}$

Presenting incorrect numbers because of a flawed study design in a scientific journal is an ethical issue that should be of concern for the Editor, the readers and the dental profession. Estimates of prevalence and incidence of complications associated with dental treatments are essential for obtaining informed consent to treat the patient; they form the basis for calculations of insurance premiums and coverage; and they influence the allocation of resources and different oral healthcare personnel in countries with public dental health care systems. It is therefore imperative that these estimates are as reliable as possible. It is especially relevant with regard to prosthodontic rehabilitation, because multiple technical alternatives are usually available with different, but frequently high, costs.

\section{Dr Goodacre has responded to the commentary on his article:}

As a subscriber, avid reader, and supporter of the journal, I appreciate all your efforts to place dental publications on a truly scientific basis. However, I believe that my papers are being interpreted as something that it was never intended to be. It is not a meta-analysis and was never meant to be and I find it interesting that it has been listed in Medline as a meta-analysis for, as the commentator has indicated, it does not qualify.
The article was designed to be a review of available literature for the purpose of identifying the types of complications that have been reported in conjunction with dental implants and to suggest trends regarding the relative frequency of these complications. It was also designed to identify areas where the available literature is weak as it relates to clinical complications. The disparity in the number of studies reporting a particular complication becomes apparent as you read the literature. Many studies do not mention certain complications and as readers we do not know whether they encountered that complication in their study and did not report it or whether they were not collecting data related to that complication.

Additionally, since there are modest differences in the percentage of implants that are successful as it relates to the type of prosthesis being used and the arch where the prosthesis is located (i.e., maxilla vs mandible in completely edentulous patients; maxillary implant overdenture $v s$ maxillary fixed complete denture), all implant studies should identify the types of prostheses used and the number of implants used with each type of prosthesis and in each arch. Unfortunately, some excellent articles do not provide such data and therefore they were not included in the review since the percentage of implants lost was specifically related to prosthesis and arch.

\section{Asbjørn Jokstad \\ Department of Dental Prosthetics and Oral Function, Faculty of Dentistry, University of Oslo, Oslo, Norway}

1. Goodacre CJ, Bernal G, Rungcharassaeng K, Kan JY. Clinical complications in fixed prosthodontics. J Prosthet Dent 2003; 90:31-41.

2. Berglundh $T$, Persson $L$, Klinge $B$. A systematic review of the incidence of biological and technical complications in implant dentistry reported in prospective longitudinal studies of at least 5 years. J Clin Periodontol 2002; 29(Suppl 3): 197-212.

3. Jokstad A, Braegger U, Brunski JB, Carr AB, Naert I, Wennerberg A. Quality of dental implants. Int Dent J 2003; 53(Suppl 2):409-443.

Evidence-Based Dentistry (2004) 5, 70-71.

doi:10.1038/sj.ebd.6400267 